\title{
A Comparative study of machine learning algorithms on thyroid disease prediction
}

\author{
${ }^{1}$ Shaik Razia, ${ }^{2}$ P. Swathi Prathyusha, ${ }^{3}$ N. Vamsi Krishna, ${ }^{4}$ N. Sathya Sumana \\ ${ }^{\text {I} A s s o c i a t e ~ P r o f e s s o r, ~ K o n e r u ~ L a k s h m a i a h ~ E d u c a t i o n ~ F o u n d a t i o n, ~ V a d d e s w a r a m, ~ G u n t u r, ~ A n d h r a ~ P r a d e s h, ~ I n d i a ~} 522502$ \\ ${ }^{2,3,4}$ UG Students, Koneru Lakshmaiah Education Foundation, Vaddeswaram, Guntur, Andhra Pradesh, India 522502 \\ *Email: razia28sk@gmail.com
}

\begin{abstract}
Thyroid illness is a medicinal state that influences the functionality of the thyroid organ that is thyroid gland [1](Guyton, 2011).The indications of thyroid ailment differ basing upon the type. There are four most common varieties: hypothyroidism (low capacity) which is caused due to the insufficiency of the thyroid hormones; hyperthyroidism (high capacity) which is caused due to the existence of the thyroid hormones more than just sufficient, basic variations from the norm, most normally an augmentation of the thyroid organ; and tumors which can be benign or can cause cancer. It is additionally conceivable to have irregular thyroid capacity tests with no clinical side effects [2](Bauer $\&$ al, 2013). In this study a comparative thyroid disease diagnosis were performed by using Machine learning techniques that is Support Vector Machine (SVM), Multiple Linear Regression, Naïve Bayes, Decision Trees. For this purpose, thyroid disease dataset gathered from the UCI machine learning database was used.
\end{abstract}

Keywords: SVM, Multiple Linear Regression, Nä̈ve Bayes, Decision Trees.

\section{Introduction}

Thyroid issues are impact on the thyroid organ, it is a butterfly shaped organ within the front of the neck.

The thyroid has basic components to coordinate totally different metabolic ways during the body. Different types of thyroid issue impact either its structure or limit.

The thyroid organ is organized beneath the Adam's apple wrapped around the trachea (windpipe). A small tissue of inside the organ called as the isthmus; attach the two thyroid projections on all sides. The thyroid utilizes iodine to convey the major hormones. Thyroxine, usually known as T4, is the essential hormone made by the organ. When transport by ways for the dissemination framework to the body's tissues, a small piece of the T4 released from the organ is modified over to triiodothyronine (T3) that is the most unique hormone.

The cerebrum includes the limit of the thyroid organ is overseen by an info framework. When thyroid hormone levels are precisely low, the hypothalamus in the cerebrum transfer a hormone known as thyrotropin releasing hormone(TRH) that causes the pituitary organ (arranged at the base of the brain) to release thyroid invigorating hormone (TSH). TSH enables the thyroid organ to release more T4.

\subsection{Dataset Description: [3]}

\begin{tabular}{|l|l|l|}
\hline Attribute & Data Type & Value Range \\
\hline Age & Real & {$[0.00,0.93]$} \\
\hline Sex & Integer & {$[0,1]$} \\
\hline On_thyroxine & Integer & {$[0,1]$} \\
\hline Query_on_thyroxine & Integer & {$[0,1]$} \\
\hline antithyroid_medication & Integer & {$[0,1]$} \\
\hline Sick & Integer & {$[0,1]$} \\
\hline Pregnant & Integer & {$[0,1]$} \\
\hline Thyroid_surgery & Integer & {$[0,1]$} \\
\hline I131_treatment & Integer & {$[0,1]$} \\
\hline Query_hypothyroid & Integer & {$[0,1]$} \\
\hline Query_hyperthyroid & Integer & {$[0,1]$} \\
\hline Lithium & Integer & {$[0,1]$} \\
\hline Goitre & Integer & {$[0,1]$} \\
\hline Tumor & Integer & {$[0,1]$} \\
\hline Hypopituitary & Integer & {$[0,1]$} \\
\hline Psych & Integer & {$[0,1]$} \\
\hline TSH & Real & {$[0.0,0.53]$} \\
\hline T3 & Real & {$[.0005,18]$} \\
\hline TT4 & Real & {$[0.0020,0.6]$} \\
\hline T4U & Real & {$[0.017,0.233]$} \\
\hline FTI & Real & {$[0.0020,0.642]$} \\
\hline Class & Integer & $\{1,2,3\}$ \\
\hline & & \\
\hline
\end{tabular}

Where class is varied as following

Normal - 1

Hyperthyroidism - 2

Hypothyroidism - 3 


\subsection{Implementation}

As the data set has no missing values at the pre-processing stage the data set is imported using the pandas library in python, and it is then splitted into training set and test set which consists of 5760 and 1440 observations respectively and they are tested on various algorithms of machine learning and the split as of randomness and the predicted values are compared and the accuracy of the model or the algorithm is calculated using the below formula

$$
\text { Accuracy }=\frac{T P+T N}{n} * 100
$$

Where TP=True Positives

$\mathrm{TN}=$ True Negatives

$\mathrm{n}=$ Number of observations in test set

To find some of true positives and true negatives we have to find the sum of principal diagonal in confusion matrix. The confusion matrix is a matrix of $n * n$ rows and columns that depend upon the elements in output class. In this case the confusion matrix is of $3 * 3$ matrix.

\section{Multiple Linear Regressions}

It is augmentation of simple linear regression. It takes the attributes as input data sources and delivers a yield.

MLR examines the connection between at least two IVs and a solitary DV, where IV is an independent variable and DV is a dependent variable.

$\mathrm{Y}=\mathrm{x} 1+\mathrm{x} 2+\ldots+\mathrm{xi}$

Where $y$ is a dependent variable which depends upon $\mathrm{x} 1, \mathrm{x} 2, \mathrm{x} 3 \ldots \ldots, \mathrm{xi}$ which are independent variables.

$\mathrm{TP}+\mathrm{TN}=1319$

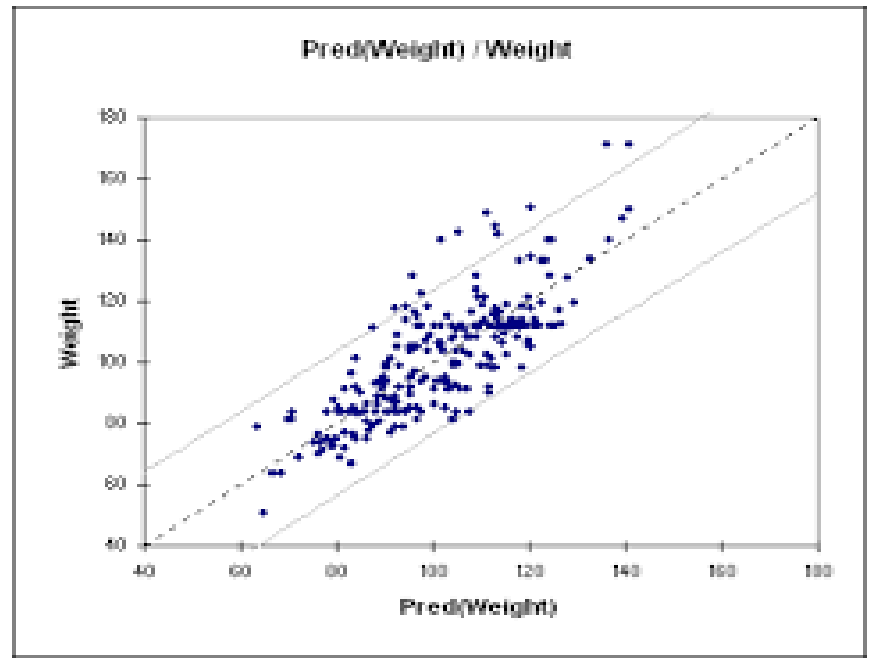

\subsection{Confusion matrix of Multiple Linear Regressions}

\begin{tabular}{|l|l|l|l|}
\hline & 0 & 1 & 2 \\
\hline 0 & 2 & 19 & 18 \\
\hline 1 & 0 & 1 & 82 \\
\hline 2 & 0 & 2 & 1316 \\
\hline
\end{tabular}

By using multi linear regression we can get the correctness of 91.59\%

\section{Svm (Support Vector Machine)}

Support Vector Machine (SVM) is a managed machine learning count which can be used for both classification and regression issues. Regardless, it's usually used as a bit of arrangement problems. During this figuring, we have a tendency to plot every data point factor as some extent in $n$-dimensional area (where $n$ is number of qualities you have) with the estimation of each half being the estimation of a selected organize. By then, we have a tendency to perform characterization by finding the hype-plane that completely different the two categories notably well.

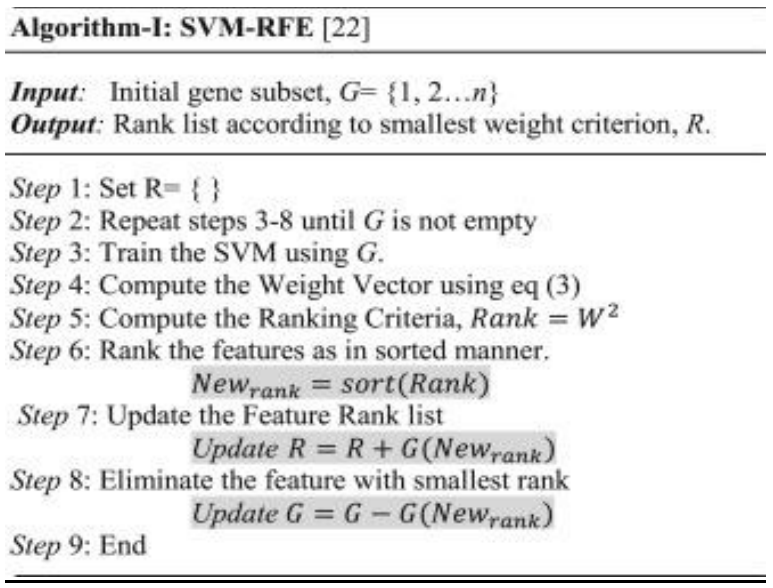

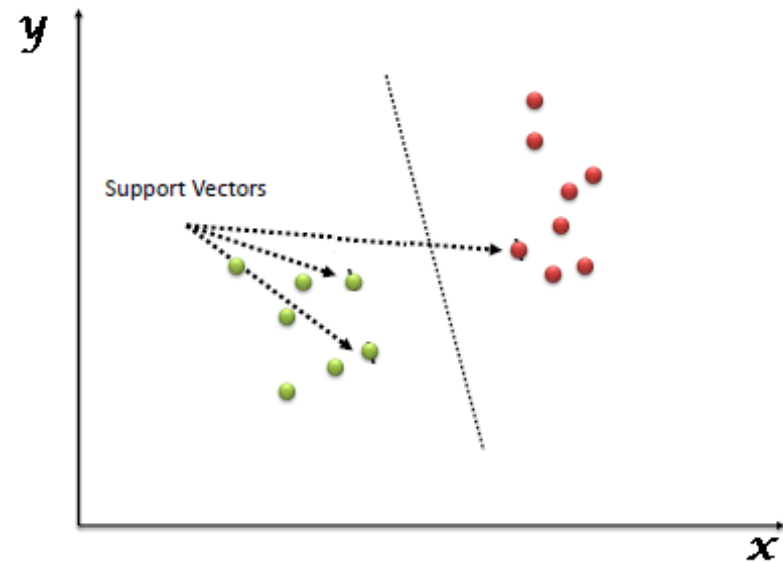

$\mathrm{TP}+\mathrm{TN}=1383$

\subsection{Confusion matrix of SVM}

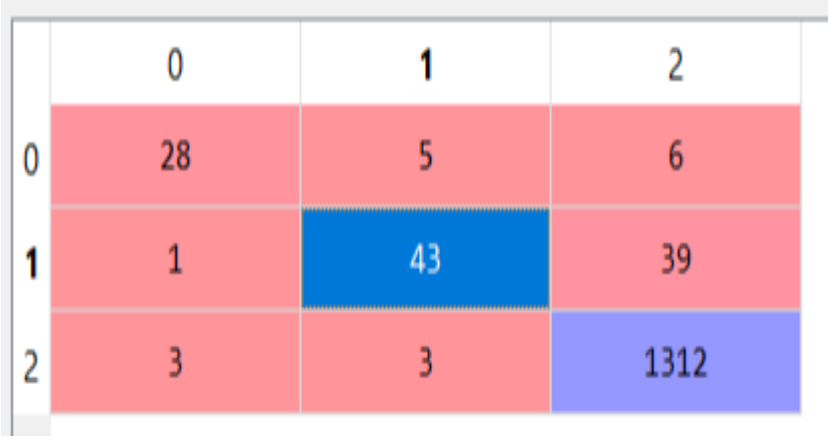

By using Support vector machine we can get the accuracy of $\mathbf{9 6 . 0 4 \%}$ 


\section{Naive Bayes}

Naive Bayes classifiers are an accumulation of classification methods based on Bayes' Theorem. It isn't a solitary algorithms yet a group of algorithms where every one of them share a typical guideline, i.e. each combination of attributes being classified is autonomous of each other.

The dataset is divided into two different categories like feature matrix and response vector.

$\rightarrow$ The feature matrix consists each and every vector that is nothing but row of dataset in which every vector comprises of the estimate of dependent features.

$\rightarrow$ Response vector contains the esteem of class variable (prediction or yield) for each vector which is nothing but a row of the feature matrix.

\subsection{Bayes Theorem}

Bayes' Theorem finds the likelihood of an event happening given the likelihood of another event that has just happened. Bayes' hypothesis is expressed numerically as the following condition:

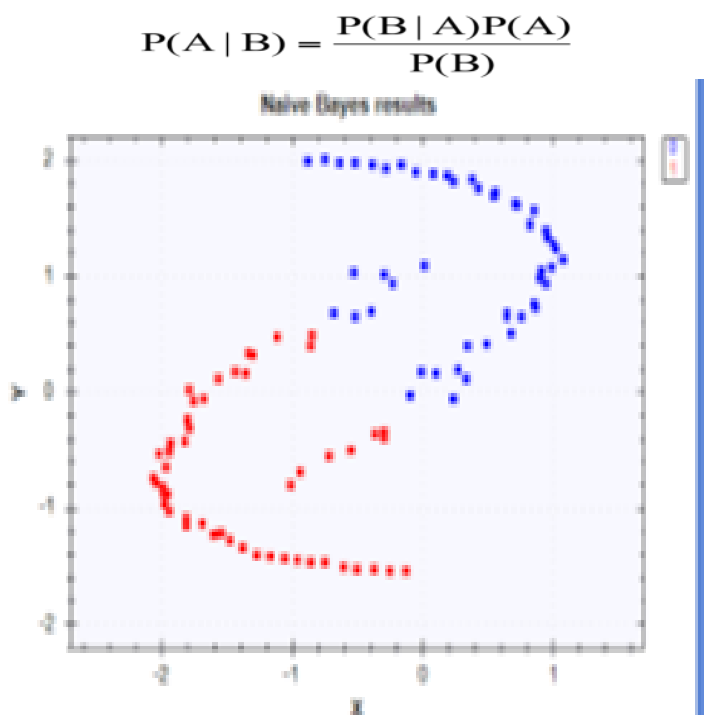

Algorithm

Step 1: Convert the data set into a frequency table. Step 2: Create Likelihood table by finding the probabilities.

Step 3: Now, use Naive Bayesian equation to calculate the posterior probability for each class. The class with the highest posterior probability is the outcome of prediction.

$\mathrm{TP}+\mathrm{TN}=91$

\subsection{Confusion matrix of Naïve Bayes}

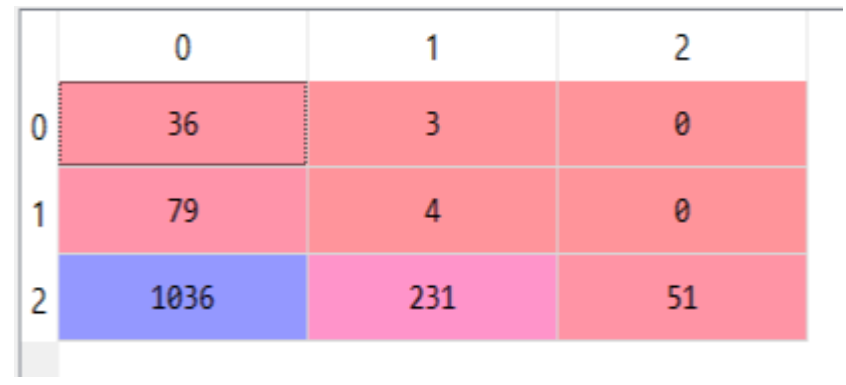

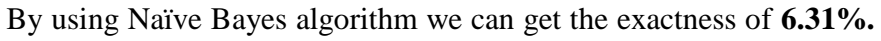
So Naïve Bayes has the least accuracy.

\section{Decision Trees}

The Decision tree is one of the classification methods. This learning calculation applies a separation and conquer methodology that can also be called as divide and conquer to build the tree. The arrangements of instances are related by an arrangement of attributes.

A Decision tree includes hubs and leaves, where hubs represent a test on the estimations of a trait and leaves represent the class of an instance that fulfills the conditions.

The result is "true or "false"e that is nothing but a categorical variable. Standards can be gotten from the way beginning from the root hub to the leaf and using the hubs on way as preconditions for the rule, to foresee the class at the leaf. The tree pruning must be done to expel pointless preconditions and duplications.

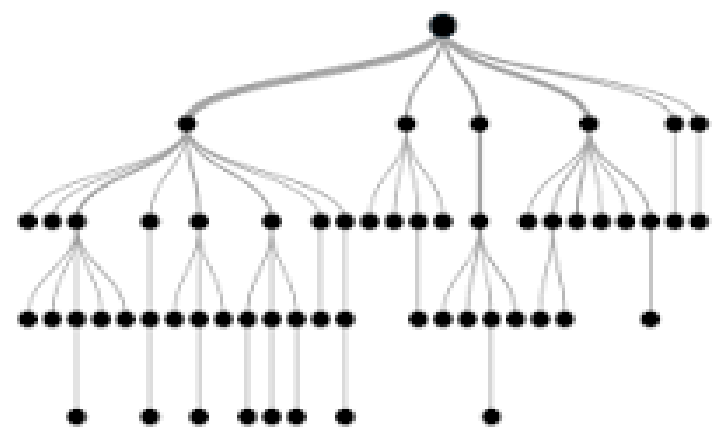

\section{Decision Tree Algorithm Pseudo code}

- Place the best attribute of the dataset at the root of the tree.

- Split the training set into subsets. ...

- Repeat step 1 and step 2 on each subset until you Find leaf nodes in all the branches of the tree.

$\mathrm{TP}+\mathrm{TN}=1429$

\section{Confusion matrix of Decision trees}

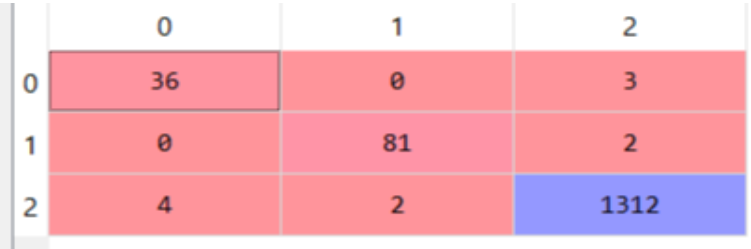

By using Decision tree algorithm we can get the highest correctness of $99.23 \%$. 


\section{Results}

\subsection{The comparison of Results}

\begin{tabular}{|l|l|}
\hline Algorithm & Accuracy \\
\hline Multiple Linear Regression & $91.59 \%$ \\
\hline SVM & $96.04 \%$ \\
\hline Naïve Bayes & $6.31 \%$ \\
\hline Decision Trees & $99.23 \%$ \\
\hline
\end{tabular}

\subsection{Multi Linear Regression output}

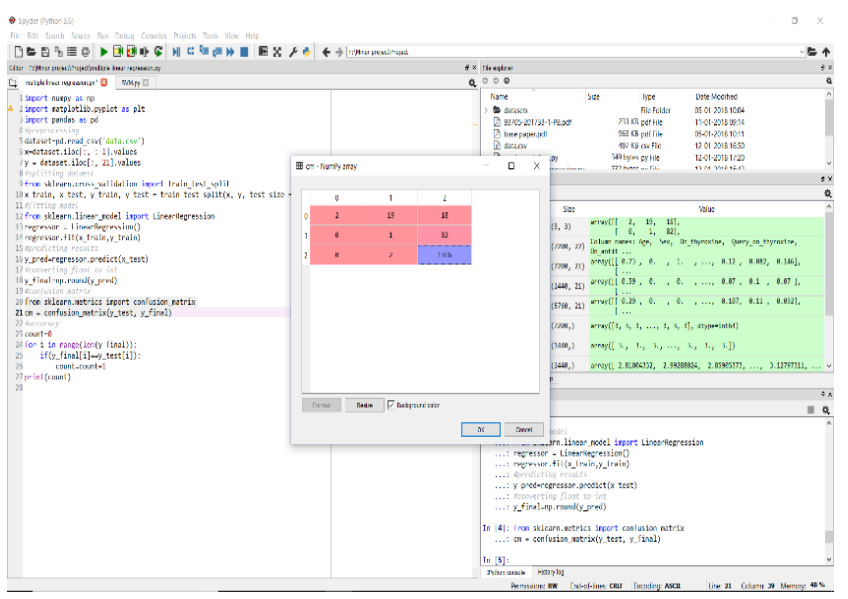

\subsection{Support Vector Machine output}

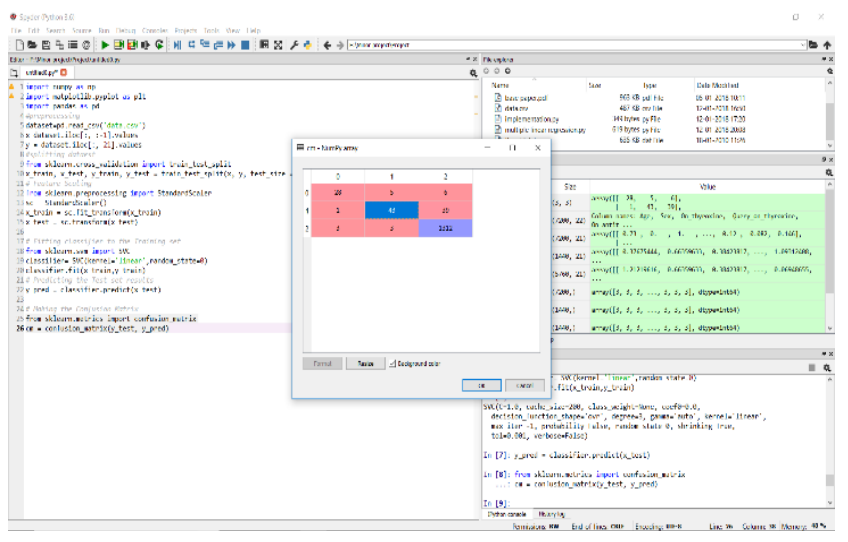

\subsection{Naïve Bayes output}

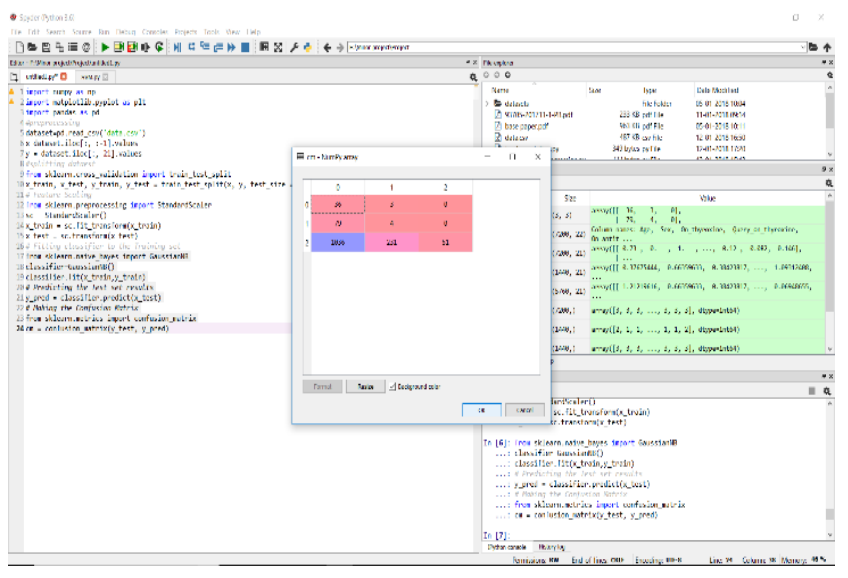

\subsection{Decision Tree output}

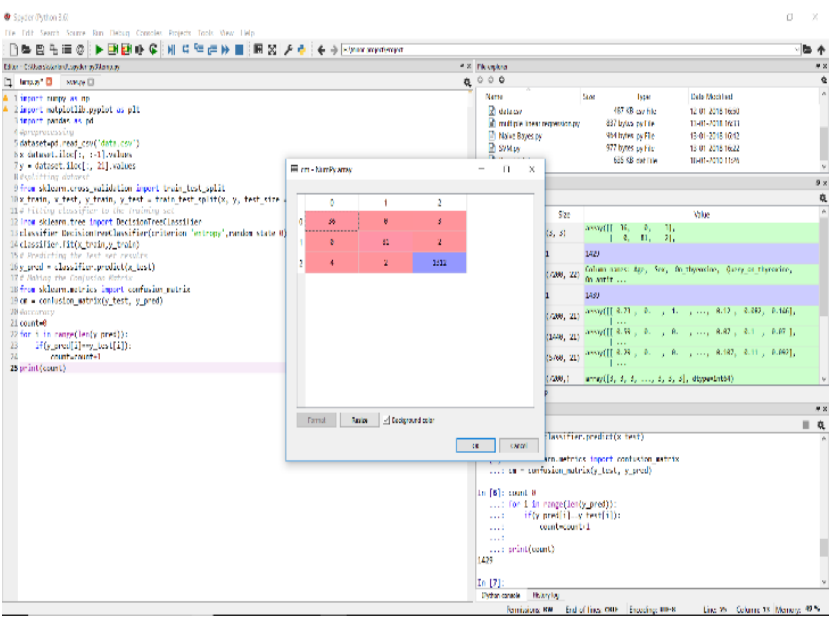

\section{Conclusion}

This paper presents a comparative study on thyroid disease diagnosis by using Support Vector Machine (SVM), Multiple Linear Regression, Naïve Bayes and Decision Trees. The results were compared and it was seen that Decision Trees could be successfully used to help the diagnosis of thyroid disease. It is observed that the Decision Trees outperformed the SVM, Multi linear regression, Naïve Bayes with respect to the accuracy of the network to diagnose the thyroid disease.

\section{References}

[1] C., Guyton, Arthur. Guyton and Hall textbook of medical physiology, OCLC 434319356

[2] Bauer, DC; et al. (2Ol3). Path physiology of Disease: An Introduction to Clinical Medicine, Seventh Edition, New York, NY: McGraw-Hill - via Access Medicine.

[3] http://sci2s.ugr.es/keel/dataset.php?cod=67

[4] Keleş A, Keleş A. Estdd: Expert system for thyroid dis-eases diagnosis. Expert Systems with Applications. 20O8; 34:242-6.

[5] Ozyilmaz L, Yildirim T. Diagnosis of thyroid diseaseusing artificial neural network methods. Proceedings of the 9th International Conference on Neural Information Processing, 2OO2. ICONIP'O2; 2OO2. p. 2O33-6.

[6] Temurtas F. A comparative study on thyroid disease diagnosis using neural networks. Expert Systems with Applications. 2009; 36:944-9.

[7] Hasan Makas, Nejat Yumusak (2ol3) A Comprehensive Study On Thyroid Diagnosis By Neural Networks And Swarm Intelligence. International Conference On Electronics, Computer And Computation (Icecco):L8o-L84.Doi:Lo.Llo9/Icecco.2ol3.6718258

[8] V. Prasad, T. Srinivasa Rao, M. Surendra Prasad Babu, Thyroid Disease Diagnosis Via Hybrid Architecture Composing Rough Data Sets Theory And Machine Learning Algorithms. Soft Computing 2o(3):L179-L189 2ol5.

[9] Li-Na Li, Ji-Hong Ouyang, Hui-Ling Chen, Da-You Liu, A Computer Aided Diagnosis System For Thyroid Disease Using Extreme Learning Machine. Journal Of Medical Systems 36(5):3327-3337 2ol2

[10] Shaik.Razia, M.R.Narasingarao Published "A Neuro Computing Frame Work For Thyroid Disease Diagnosis Using Machine Learning Techniques", Vol.95. No.9. Pages L996-2oo5, Issn: L9928645.

[11] P. Gopi Krishna, K. Sreenivasa Ravi "Designing A Multipurpose Reconfigurable Wireless Node For Broadcasting And Unicasting In Rereal Time Applications" In International Journal Of Pure And Applied Mathematics (Ijpam). Volume L15 No. 8 2ol7, 5o5-5lo.

[12] P Gopi Krishna, K Sreenivasa Ravi "Implementation Of Mqtt Protocol On Low Resourced Embedded Network" In International 
Journal Of Pure And Applied Mathematics (Ijpam). Volume L16 No. 6 2ol7, L61-L66.

[13] Dr. Seetaiah Kilaru, Hari Kishore K, Sravani T, Anvesh Chowdary L, Balaji $\mathrm{T}$ "Review And Analysis Of Promising Technologies With Respect To Fifth Generation Networks", 2ol4 First International Conference On Networks \& Soft Computing, Issn:978-L-4799-34867/L4,Pp.27o-273,August2ol4.

[14] N.Prathima, K.Hari Kishore, "Design Of A Low Power And High Performance Digital Multiplier Using A Novel 8t Adder", International Journal Of Engineering Research And Applications, Issn: 2248-9622, Vol. 3, Issue.L, Jan-Feb., 2 ol3.

[15] T. Padmapriya And V. Saminadan, "Improving Throughput For Downlink Multi User Mimo-Lte Advanced Networks Using Sinr Approximation And Hierarchical Csi Feedback", International Journal Of Mobile Design Network And Innovation- Inderscience Publisher, Issn : L744-285o Vol. 6, No.L, Pp. L4-23, May 2015.

[16] S.V.Manikanthan And K. Srividhya "An Android Based Secure Access Control Using Arm And Cloud Computing", Published In: Electronics And Communication Systems (Icecs), 2ol5 2nd International Conference On 26-27 Feb. 2015,Publisher: Ieee, Doi: Lo.Llo9/Ecs.2ol5.7124833.

[17] M. Rajesh, Manikanthan, "Annoyed Realm Outlook Taxonomy Using Twin Transfer Learning”, International Journal Of Pure And Applied Mathematics, Issn No:L314-3395, Vol-L16, No. 21, Oct 2 ol7.

[18] [18] K.Srikar, M.Akhil, V.Krishna Reddy "Execution Of Cloud Scheduling Algorithms" International Innovative Research Journal Of Engineering And Technology Issn No: 2456-L983.Volume 2, Issue 4 June 2017.

[19] Meherwar Fatima, M. P. (2ol7). Survey Of Machine Learning Algorithms For Disease Diagnostic. Journal Of Intelligent Learning Systems And Applications, L-L6.

[20] Jian, A. (2ol5). Machine Learning Techniques For Medical Diagnosis. Icstat.

[21] Alic, B. (2ol7). Machine Learning Techniques For Classification Of Diabetes And Cardiovascular Diseases. Mediterranean Conference On Embedded Computing.

[22] Shaik Razia, M.R.Narasingarao, Polaiah Bojja Published "Development And Analysis Of Support Vector Machine Techniques For Early Prediction Of Breast Cancer And Thyroid" In Scopus Indexed Journal Jardcs (Journal Of Advanced Research In Dynamical And Control Systems, 2017.Vol.9.Sp.Issue:6 Issn: 1943023x Page No: 869-878).

[23] Shaik Razia Published "A Review On Disease Diagnosis Using Machine Learning Techniques" International Journal Of Pure And Applied Mathematics, 2017, Volume 117, No. 16 2017, 79-85, Issn: 1311-8080.

[24] Shaik Razia, M.R.Narasingarao, Polaiah Boija Published "The Analysis Of Data Representation Techniques For Early Prediction Of Breast Cancer" International Journal Of Pure And Applied Mathematics, 2017, Volume-115, Issue: 6, Issn: 1311-8080, Issn: 1314-3395 Page No: 177-183.

[25] Shaik Razia, M.R.Narasingarao Published "Machine Learning Techniques For Thyroid Disease Diagnosis - A Review" Indian Journal Of Science And Technology, Issn: 09746846, Volume-9, Issue 28, July 2016.

[26] Shaik Razia, M.R.Narasingarao,G R Sridhar Published "A Decision Support System For Prediction Of Thyroid Disease- A Comparison Of Multilayer Perception Neural Network And Radial Basis Function Neural Network" Journal Of Theoretical And Applied Information Technology, 31st October 2015. Vol.80. No.3, Issn: 1992-8645.

[27] Meka Bharadwaj, Hari Kishore "Enhanced Launch-Off-Capture Testing Using Bist Designs" Journal Of Engineering And Applied Sciences, Issn No: 1816-949x, Vol No.12, Issue No.3, Page: 636643, April 2017.

[28] P Bala Gopal, K Hari Kishore, R.R Kalyan Venkatesh, P Harinath Mandalapu "An Fpga Implementation Of On Chip Uart Testing With Bist Techniques", International Journal Of Applied Engineering Research, Issn 0973-4562, Volume 10, Number 14 , Pp. 34047-34051, August 2015. 